\title{
CURIE TEMPERATURE OF AMORPHOUS FERROMAGNETS OF TYPE Fe-TM-M
}

\author{
E. NOWAKOWSKA \\ Department of Physics, Agriculture University SGGW \\ Rakowiecka 26/30, 02-528 Warszawa, Poland \\ J. Oleniacz, W. Zycir and W. Dudek \\ Institute of Physics, Warsaw University of Technology \\ Koszykowa 75, 00-662 Warszawa, Poland \\ (Received July 10, 1996; revised version May 28, 1997)

\begin{abstract}
The theoretical Ifeisenberg model of an amorphous ferromagnet having two magnetic components in the Bethe-Peierls-Weiss approximation was applied to study the concentration dependence of the Curie temperature of the Fe-TM-M alloys (TM - transition metal, M - metalloid). The analysis of the experimental data in comparison with the model calculations was performed.
\end{abstract}

PACS numbers: $75.50 . \mathrm{Kj}, 76.80 .+\mathrm{y}, 75.50 . \mathrm{Bb}$

\section{Introduction}

Experimental data show that the Curie temperatures $\left(T_{\mathrm{C}}\right)$ of transition metal amorphous alloys follow the same trends but are systematically lower than the Curie temperatures of crystalline alloys. It is also known that metal and metalloid variations strongly affect the Curie temperature of transition metal amorphous alloys. These facts can be explained as the effects of chemical environment and structural disorder.

The Curie temperature has been measured for a large number of transition metal amorphous alloys. Figure 1 shows the concentration dependence of the Curie temperature for the $\left(\mathrm{Fe}_{1-c} \mathrm{TM}_{c}\right)_{80} \mathrm{~B}_{20}(\mathrm{TM}=\mathrm{Co}, \mathrm{Ni}, \mathrm{Mn}, \mathrm{Cr}, \mathrm{Ti}, \mathrm{V})$ amorphous alloys published by us previously [1]. Because these experimental data are restricted to low TM concentration, other experimental data in a wider concentration range and with other glass-forming elements are presented as well [2-6].

Some qualitative features of the magnetic interaction are evident from the observed concentration dependence of $T_{\mathrm{C}}$. The high values of the Curie temperature of the Fe-rich samples indicate that the $\mathrm{Fe}-\mathrm{Fe}$ exchange $J_{\mathrm{FeFe}}$ is ferromagnetic 


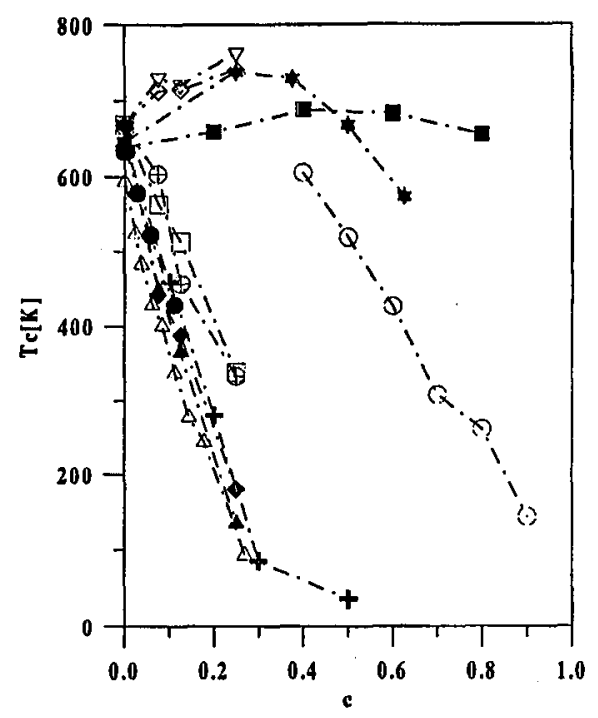

Fig. 1. Curie temperatures of transition metal-metalloid alloys as a function of transition metal composition. Dashed lines follow the data. $\diamond\left(\mathrm{Fe}_{1-c} \mathrm{Ni}_{c}\right)_{80} \mathrm{~B}_{20}$, $\nabla\left(\mathrm{Fe}_{1-c} \mathrm{Co}_{c}\right)_{80} \mathrm{~B}_{20}, \square\left(\mathrm{Fe}_{1-c} \mathrm{Mn}_{c}\right)_{80} \mathrm{~B}_{20}$, full triangle $\left(\mathrm{Fe}_{1-c} \mathrm{Cr}_{c}\right)_{80} \mathrm{~B}_{20}$, full diamond $\left(\mathrm{Fe}_{1-c} \mathrm{~V}_{c}\right)_{80} \mathrm{~B}_{20}, \quad \oplus \quad\left(\mathrm{Fe}_{1-c} \mathrm{Ti}_{c}\right)_{80} \mathrm{~B}_{20} \quad[1], \quad \mathrm{O} \quad\left(\mathrm{Fe}_{1-c} \mathrm{Ni}_{c}\right)_{75} \mathrm{Si}_{15} \mathrm{~B}_{10} \quad$ [2], - $\left(\mathrm{Fe}_{1-c} \mathrm{Co}_{c}\right)_{75} \mathrm{P}_{16} \mathrm{~B}_{6} \mathrm{Al}_{3}$ [3], + $\left(\mathrm{Fe}_{1-c} \mathrm{Mn}_{c}\right)_{75} \mathrm{P}_{16} \mathrm{~B}_{6} \mathrm{Al}_{3}$ [4], * $\left(\mathrm{Fe}_{1-c} \mathrm{Ni}_{c}\right)_{80} \mathrm{~B}_{20}$ [5], $\Delta\left(\mathrm{Fe}_{1-c} \mathrm{Cr}_{c}\right)_{86} \mathrm{~B}_{14}, \bullet\left(\mathrm{Fe}_{1-c} \mathrm{~V}_{c}\right)_{80} \mathrm{~B}_{20}[6]$.

and large. In the case of $\mathrm{Fe}-\mathrm{Ni}$ and $\mathrm{Fe}-\mathrm{Co}$ amorphous alloys a maximum at a certain intermediate concentration is observed, which may be interpreted assuming that $\mathrm{Fe}-\mathrm{Ni}$ or $\mathrm{Fe}-\mathrm{Co}$ interaction is greater than that between the same species of atoms. For $\mathrm{Fe}-\mathrm{Co}$ alloys the experimental data vary relatively slowly with Co concentration and suggest that $J_{\mathrm{FeCo}}>J_{\mathrm{CoCo}}>J_{\mathrm{FeFe}}$. In the $\mathrm{Fe}-\mathrm{Ni}$ samples, the Curie temperature drops rapidly for decreasing Fe content. This is consistent with the assumption that $J_{\mathrm{FeNi}}>J_{\mathrm{FeFe}}$ and $J_{\mathrm{NiNi}}=0$. Unlike the $\mathrm{Fe}-\mathrm{Co}$ and $\mathrm{Fe}-\mathrm{Ni}$ samples, in the $\mathrm{Fe}-\mathrm{Mn}, \mathrm{Fe}-\mathrm{Cr}, \mathrm{Fe}-\mathrm{V}, \mathrm{Fe}-\mathrm{Ti}$ alloys a large linear decrease in $T_{\mathrm{C}}$ is observed. This unusual behaviour is due to the presence of an antiferromagnetic exchange interaction. The samples with low Fe concentrations, where competing ferromagnetic and antiferromagnetic interaction occur, show spin-glass-like characteristics. In order to obtain more quantitative information regarding the exchange interactions, one can compare the observed data with theoretical results using various models. A number of effective field approaches have been advanced to interpret the experimental data. The simplest theory which can explain the transition from the ordered ferromagnetic pliase to the disordered paramagnetic phase is the molecular field approximation (MFA). Moreover, the MFA cannot be used to study the onset of ferromagnetism in amorphous solids since it does not predict a critical concentration. Another objection to the MFA in amorphous ferromagnets lies in the absence of the effect of fluctuations in exchange interactions on $T_{\mathrm{C}}$. Therefore, it is necessary to investigate the effect of disorder within the framework 
of other effective field methods. The disordered composition dependent exchange interaction can be treated in a coherent potential approximation (CPA). In the weak scattering limit the CPA reduces to the results of the mean field theory. Ilowever, in the strong scattering limit, the CPA predicts such effects as a critical concentration for the appearance of ferromagnetism which is beyond the scope of the mean field theory.

In amorphous materials a profound change of magnetic properties, in comparison with those for the related crystalline ones, may occur due to the fluctuations of the number of nearest neighbours around their mean value, which can be equal to the related crystalline one. The theoretical background of this problem has been studied within the framework of the Bethe-Peierls-Weiss (BPW) approximation for a classical Heisenberg ferromagnet.

\section{Results and discussion}

To study the concentration dependence of the Curie temperature we applied the theoretical IIeisenberg model of an amorphous ferromagnet of the type $\mathrm{A}_{1-c} \mathrm{~B}_{c}$ having two magnetic components in the Bethe-Peierls-Weiss approximation for classical spins, developed by Świderczak et al. [7]. This model is the extension of the calculations performed by Bobak [8] and Maksymowicz [9] on amorphous ferromagnets with one component magnetic and the other nonmagnetic. In the paper of Świderczak et al. only theoretical possibility of the model has been presented. In this paper we try to use this model to explain experimentally observed concentration dependence of the Curie temperature. We choose this method of calculations because it allows us to investigate the atomic short range order effect on magnetic properties of $\mathrm{Fe}-\mathrm{TM}-\mathrm{M}$ amorphous alloys.

The parameters of the model are: $J_{\mathrm{AA}}, J_{\mathrm{AB}}, J_{\mathrm{BB}}-$ exchange integrals for the pairs of nearest neighbours $\mathrm{AA}, \mathrm{BB}, \mathrm{AB}$ respectively, $S_{\mathrm{A}}, S_{\mathrm{B}}$ - spins for the $\mathrm{A}$ and $\mathrm{B}$ atoms respectively, $\left\langle Z_{\mathrm{A}}\right\rangle,\left\langle Z_{\mathrm{B}}\right\rangle$ - mean values of the coordination numbers $Z_{\mathrm{A}}, Z_{\mathrm{B}}$ around $\mathrm{A}$ atoms and $\mathrm{B}$ atoms respectively (both averages are taken over all atoms of the same kind), $\delta$ - parameter which takes into account the differences between the mean values of the coordination number $Z_{\mathrm{A}}$ and $Z_{\mathrm{B}}$ around $A$ and $B$ atoms respectively defined as

$$
\delta=\frac{\left\langle Z_{\mathrm{A}}\right\rangle-\left\langle Z_{\mathrm{B}}\right\rangle}{\left\langle Z_{\mathrm{A}}\right\rangle}
$$

$\Delta_{\mathrm{A}}^{2}, \Delta_{\mathrm{B}}^{2}-$ mean square fluctuation of the coordination number

$$
\Delta_{\mathrm{A}}^{2}=\frac{\left\langle\left(Z_{\mathrm{A}}-\left\langle Z_{\mathrm{A}}\right\rangle\right)^{2}\right\rangle}{\left\langle Z_{\mathrm{A}}\right\rangle^{2}}
$$

and similarly for $\mathrm{B}$ atoms $\left(\Delta_{\mathrm{N}}^{2}=0\right.$ for crystalline system; for amorphous alloy $\Delta_{\mathrm{N}}^{2}$ varies from 0.01 to 0.1$), \alpha$ - Cowley's parameter which reflects deviations from the complex disorder, when all the atoms are distributed randomly, $\alpha$ is defined as

$$
\alpha=1-\frac{\left\langle Z_{\mathrm{AB}}\right\rangle}{\left\langle Z_{\mathrm{AB}}^{*}\right\rangle}
$$

$\left\langle Z_{\mathrm{AB}}\right\rangle$ is the mean number of $\mathrm{B}$ atoms in the nearest neighbour coordination shell of A-type atom and $\left(Z_{\mathrm{AB}}^{*}\right)$ is the same number in the completely random system. 
Positive $\alpha$ describes tendency to clustering of atoms of the same kind and has the maximum value $\alpha=1$, negative $\alpha$ describes tendency to clustering $A-B$ pairs (chemical ordering), the lower bound for $\alpha=\alpha_{\min }$ is given in Ref. [7], $\alpha=0$ describes a fully disordered system. We expect the values $\Delta_{\mathrm{N}}^{2}=0.1$ and $\alpha=\alpha_{\min }$ to be consistent with typical conditions in some amorphous alloys [9].

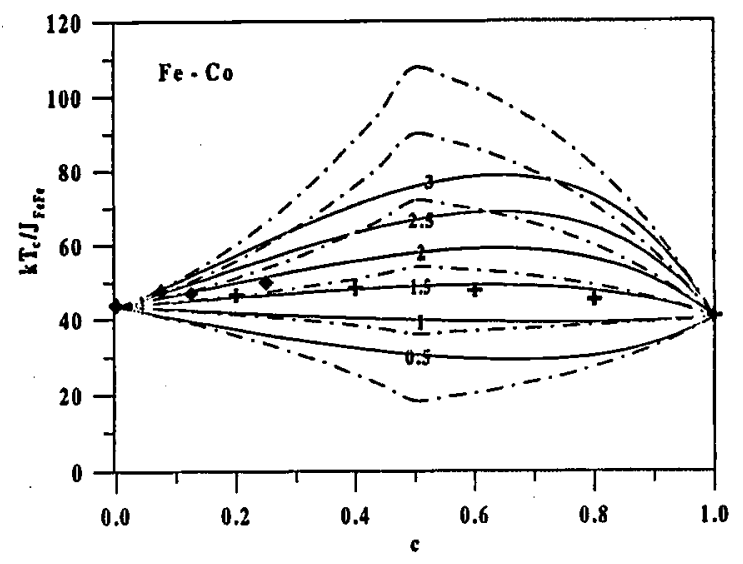

Fig. 2. Curie temperature for Fe-Co alloys as a function of transition metal concentration. Solid lines are the model calculations for $\alpha=0$, dashed lines - for $\alpha=\alpha_{\min }$, full diamond $\left(\mathrm{Fe}_{1-c} \mathrm{Co}_{c}\right)_{80} \mathrm{~B}_{20}[1],+\left(\mathrm{Fe}_{1-c} \mathrm{Co}_{c}\right)_{75} \mathrm{P}_{16} \mathrm{~B}_{6} \mathrm{Al}_{3}$ [3].

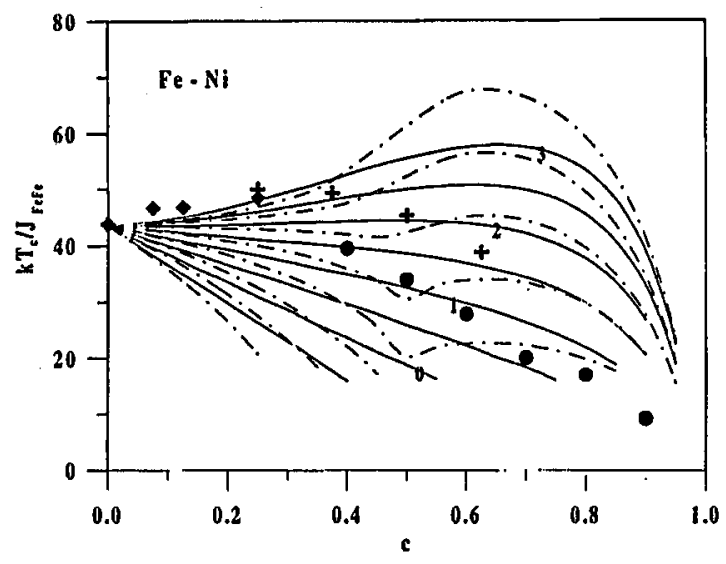

Fig. 3. Curie temperature for Fe-Ni alloys as a function of transition metal concentration. Solid lines are the model calculations for $\alpha=0$, dashed lines - for $\alpha=\alpha_{\min }$, full diamond $\left(\mathrm{Fe}_{1-c} \mathrm{Ni}_{c}\right)_{80} \mathrm{~B}_{20}[1], \bullet\left(\mathrm{Fe}_{1-c} \mathrm{Ni}_{c}\right)_{75} \mathrm{Si}_{15} \mathrm{~B}_{10}$ [2], +( $\left.\mathrm{Fe}_{1-c} \mathrm{Ni}_{c}\right)_{80} \mathrm{~B}_{20}$ [5]. 


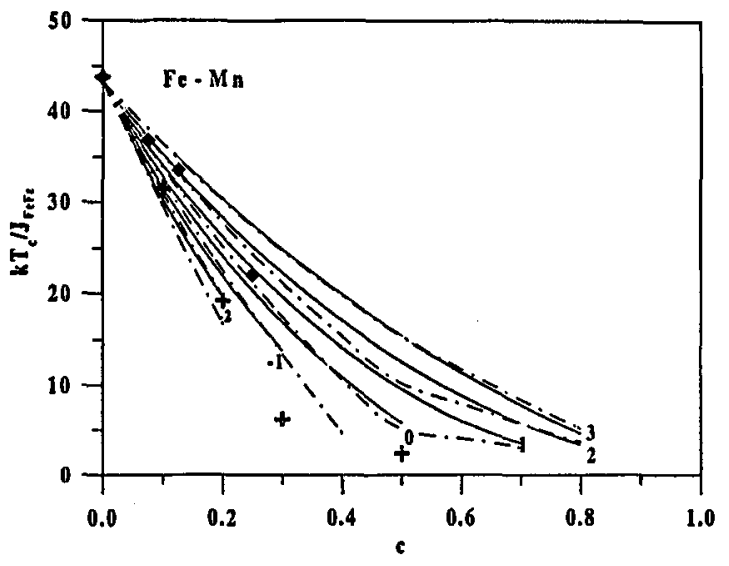

Fig. 4. Curie temperature for Fe-Mn alloys as a function of transition metal concentration. Solid lines are the model calculations for $\alpha=0$, dashed lines - for $\alpha=\alpha_{\min }$, full diamond $\left(\mathrm{Fe}_{1-c} \mathrm{Mn}_{c}\right)_{80} \mathrm{~B}_{20}[1],+\left(\mathrm{Fe}_{1-c} \mathrm{Mn}_{c}\right)_{75} \mathrm{P}_{16} \mathrm{~B}_{6} \mathrm{Al}_{3}$ [4].

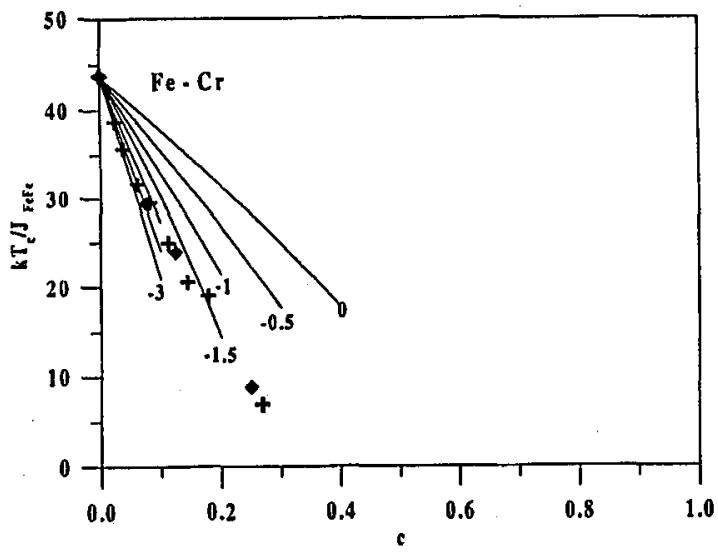

Fig. 5. Curie temperature for $\mathrm{Fe}-\mathrm{Cr}$ alloys as a function of transition metal concentration. Solid lines are the model calculations for $\alpha=0$, full diamond $\left(\mathrm{Fe}_{1-c} \mathrm{Cr}_{c}\right)_{80} \mathrm{~B}_{20}[1]$, $+\left(\mathrm{Fe}_{1-c} \mathrm{Cr}_{c}\right)_{86} \mathrm{~B}_{14}[6]$.

Various combinations of these parameters were used in our calculations. It was established that the results do not depend essentially on the values of $\Delta_{\mathrm{N}}^{2}$ (plots of $T_{\mathrm{C}}$ are consistent to within $1 \%$ for $\Delta_{\mathrm{N}}^{2}=0$ and $\Delta_{\mathrm{N}}^{2}=0.1$ ), so only one value $\Delta_{\mathrm{N}}^{2}=0.1$ was taken. The atomic short-range order was considered in the two limiting cases, $\alpha=0$ for a random system and $\alpha=\alpha_{\min }$ for fully ordered one. The calculations were performed for one value of $\delta=0$, this means $Z_{\mathrm{A}}=Z_{\mathrm{B}}=12$. Spin $S_{\mathrm{Fe}}=2$, exchange integral $J_{\mathrm{FeFe}}=1$ (scaled to agree with the experimental point $c=0$ ). The other parameters such as $S_{\mathrm{B}}, J_{\mathrm{FeB}}, J_{\mathrm{BB}}$ are different for different systems. Figures 2-7 show application of the model to the Fe-TM-M alloys. All 


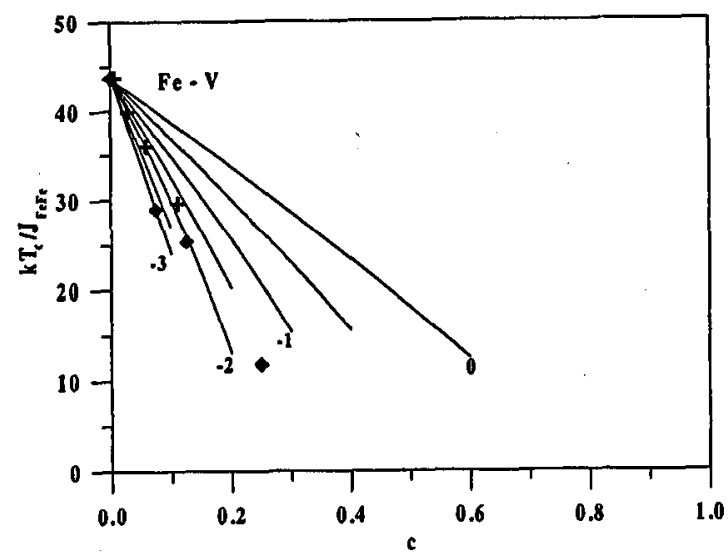

Fig. 6. Curie temperature for Fe-V alloys as a function of transition metal concentration. Solid lines are the model calculations for $\alpha=0$, full diamond $\left(\mathrm{Fe}_{1-c} \mathrm{~V}_{c}\right)_{80} \mathrm{~B}_{20}$ [1], $+\left(\mathrm{Fe}_{1-c} \mathrm{~V}_{c}\right)_{80} \mathrm{~B}_{20}[6]$.

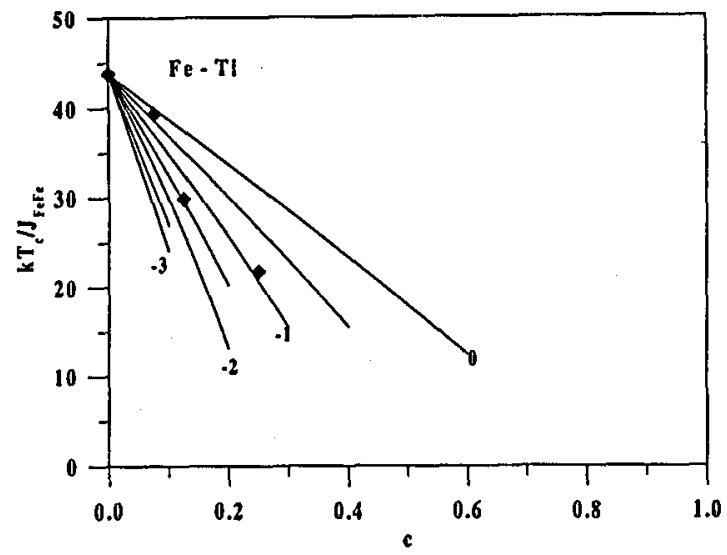

Fig. 7. Curie temperature for Fe-Ti alloys as a function of transition metal concentration. Solid lines are the model calculations for $\alpha=0$, full diamond $\left(\mathrm{Fe}_{1-c} \mathrm{Ti}_{c}\right)_{80} \mathrm{~B}_{20}$ [1].

figures show the concentration dependence of $T_{\mathrm{C}}$. Points are the experimental data. Solid lines are the predictions of the model for $\alpha=0$, dashed lines in Fig. 2-4 for $\alpha=\alpha_{\min }$. The theoretical calculations were performed for different values of exchange interaction $J_{\mathrm{FeB}}$. It was established that the variation of exchange integral $J_{\mathrm{FeB}}$ causes interesting changes in the Curie temperature against concentration plot. The influence of short range ordering on $T_{\mathrm{C}}$ is also observed. For $\alpha=0, T_{\mathrm{C}}$ is a quite linear function of concentration $c$ of the B component. The shape of this curve changes drastically when $\alpha \neq 0$, it is no more linear and a local extremum is observed (Figs. 2, 3). For Fe-Mn (Fig. 4) the effect of atomic short-range order is small and reduces to a translation. The same relation is obtained for $\mathrm{Fe}-\mathrm{Cr}, \mathrm{Fe}-\mathrm{V}$, 
Fe-Ti amorphous alloys and the results of the calculations for $\alpha=\alpha_{\min }$ are not shown in Figs. 5-7.

For Fe-Co system (Fig. 2) $S_{\mathrm{Co}_{\mathrm{o}}}=1, J_{\mathrm{CoCo}}=2.8$ to agree with the Curie temperature of the experimental point $c=1, J_{\mathrm{FeCo}}=X J_{\mathrm{FeFe}}$, where $X=0.5 \div 3$ with increment 0.5 ( $X$ is the number written on the solid curve). Good agreement of theoretical calculations and experimental data is observed. The experimental points agree with the results of the calculations $X=1.5$, this means $J_{\mathrm{FeCo}}=$ 1.5 $J_{\mathrm{FeFe}}$. The curves $X=1.5$ for $\alpha=0$ and $\alpha=\alpha_{r m m i n}$ are almost identical. The significant differences between the curves for $\alpha=0$ and $\alpha=\alpha_{\min }$ can be observed when $X \neq 1.5$.

For $\mathrm{Fe}-\mathrm{Ni}$ system (Fig. 3) $S_{\mathrm{Ni}}=0.5, J_{\mathrm{NiNi}}=0, J_{\mathrm{FeNi}}=X J_{\mathrm{FeFe}}$ where $X=-0.5 \div 3$ with increment 0.5 . Contrary to alloys with Co, $J_{\mathrm{FeNi}}$ do not remain constant for different $\mathrm{Ni}$ concentrations. For low values of $c$ the experimental data agree with the curve $X=3$. For higher concentrations the experimental values decrease more than the theoretical calculations. In this case the model calculations are not completely consistent with the experimental data, in particular for $\alpha=\alpha_{\min }$. The theoretical calculations of exchange interactions were performed in Ref. [10]. By fitting the measured values of $T_{\mathrm{C}}$ to the results of a coherent potential approximation, exchange interaction $J_{\mathrm{FeNi}}=1.3 J_{\mathrm{FeFe}}, J_{\mathrm{NiNi}}=0$ and critical concentration of $\mathrm{Fe}$ for the appearance of ferromagnetism $x_{\mathrm{c}}=0.1$ were estimated.

For $\mathrm{Fe}-\mathrm{Mn}$ system (Fig. 4) $S_{\mathrm{Mn}}=2.5, J_{\mathrm{MnMn}}=0.01, J_{\mathrm{FeMn}}=$ $X \sqrt{J_{\mathrm{FeFe}} J_{\mathrm{MnMn}}}$, where $X=-3, \div 2$ with increment 1 . Good agreement with experimental data was obtained for positive value of $J_{\mathrm{MnMn}}$. Atoms of Mn interact usually antiferromagnetically $\left(J_{\mathrm{MnMn}}<0\right)$ but in some alloys they can interact ferromagnetically, so we put exchange integral $J_{\mathrm{MnMn}}$ positive to the calculations. The effect of metalloid type on exchange interaction in amorphous alloys is observed. The experimental points for $\left(\mathrm{Fe}_{1-c} \mathrm{Mn}_{c}\right)_{80} \mathrm{~B}_{20}$ agree with the curve $X=1$ and suggest the ferromagnetic coupling of $\mathrm{Fe}$ and $\mathrm{Mn}$ ( $J_{\mathrm{FeMn}}$ is positive). In the case of $\left(\mathrm{Fe}_{1-c} \mathrm{Mn}_{\mathrm{c}}\right)_{75} \mathrm{P}_{16} \mathrm{~B}_{6} \mathrm{Al}_{3}$ experimental points suggest antiferromagnetic coupling of $\mathrm{Fe}$ and $\mathrm{Mn}\left(X=-2, J_{\mathrm{FeMn}}\right.$ is negative). The values of $J_{\mathrm{FeMn}}$ depend on the kind of metalloid. $J_{\mathrm{FeMn}}$ decreases as boron is replaced by other metalloids. The existence of critical concentration is evident. The curves with experimental data finish for $c<0.5$.

For Fe-Cr alloys (Fig. 5) $S_{\mathrm{Cr}}=1, J_{\mathrm{CrCr}}=-2, J_{\mathrm{FeCr}}=\mathrm{XJ}_{\mathrm{FeFe}}$, for $\mathrm{Fe}-\mathrm{V}$ alloys (Fig. 6) $S_{\mathrm{V}}=0.5, J_{\mathrm{VV}}=-2, J_{\mathrm{FeV}}=X J_{\mathrm{FeFe}}$, for Fe-Ti alloys (Fig. 7) $S_{\mathrm{Ti}}=0.5, J_{\mathrm{TiTi}}=-2, J_{\mathrm{FeTi}}=X J_{\mathrm{FeFe}}$. In the case of $\mathrm{Fe}-\mathrm{Cr}, \mathrm{Fe}-\mathrm{Ti}$, and $\mathrm{Fe}-\mathrm{V}$ the calculations were performed for negative values of $J_{\mathrm{FeB}}(X=-3 \div 0$ with increment 0.5 ). The experimental data suggest the antiferromagnetic interaction of $\mathrm{Cr}, \mathrm{Ti}, \mathrm{V}$ with $\mathrm{Fe}$.

On the basis of Figs. 2-4 one can conclude that the experimental data of the concentration dependence of $T_{\mathrm{C}}$ in transition metal base amorphous alloys do not strongly reflect the effects of structural disorder. The accuracy of derivative thermal magnetogravimetry (DTMG) technique [11] applied by us to the measurements of $T_{\mathrm{C}}$ is sufficient to show irregularities obtained from the model calculations for $\alpha=\alpha_{\min }$, but $T_{\mathrm{C}}$ obtained from experiment is a smooth function of alloy composition over the entire range like the model calculations for $\alpha=0$. 
In summary, it appears that the results of comparison of the experimental data with the model calculations do not provide unambiguous answers concerning effects of structural and chemical disorder on $T_{\mathrm{C}}$ in the amorphous alloys of the type Fe-TM-M. In this regard the method of calculations goes not $s \_$far from the typical molecular field approximation. The above analysis allows us to formulate only the qualitative conclusions about exchange interactions. In the case of $\mathrm{Fe}-\mathrm{Co}$ the exchange interaction of $\mathrm{Fe}$ and $\mathrm{Co}$ is ferromagnetic and strong. For $\mathrm{Fe}-\mathrm{Mn}$ the kind of interaction (ferromagnetic or antiferromagnetic) depends on the composition of the alloy. $\mathrm{Cr}, \mathrm{Ti}, \mathrm{V}$ interact aniferromagnetically with $\mathrm{Fe}$. In the case of $\mathrm{Fe}-\mathrm{Ni}$ a more complex coupling is evident.

\section{References}

[1] E. Nowakowska, W. Dudek, J. Oleniacz, W. Zych, Acta Phys. Pol. A 86, 1027 (1994).

[2] R. Moskalewicz, W. Zych, in: Digests of the Badgastein Conference Soft Magnetic Materials 8, 1987, University of Technology, Inst. of Appl. \& Techn. Physics, Vienna 1987.

[3] C.L. Chien, J.H. Hsu, P.J. Viccaro, B.D. Dunlap, G.K. Shenoy, H.S. Chen, J. Appl. Phys. 52, 1750 (1981).

[4] C.L. Chien, J.H. Hsu, J.P. Stokes, A.N. Bloch, H.S. Chen, J. Appl. Phys. 50, 11 (1979).

[5] A. Iuone, T. Masumoto, Sci. Rep. RITU 29, 305 (1981).

[6] J. Kovac, L. Potocky, L. Novak, Acla Phys. Slov. 40, 232 (1980).

[7] K. Świderczak, A. Zagórski, J. Król, Phys. Status Solidi B 134, 581 (1986).

[8] A. Bobak, Phys. Status Solidi B 109, 161 (1982).

[9] A.Z. Maksymowicz, Phys. Status Solidi B 114, 125 (1982); Phys. Status Solidi B 122,519 (1984).

[10] J.J. Becker, F.E. Luborsky, J.L. Walter, IAEE Trans. MAG-13, 988 (1977).

[11] R. Moskalewicz, Thermochim. Acta 28, 229 (1979). 